МИХАЛЬЧЕНКОВА Наталья Алексеевна - доктор политических наук, руководитель Арктического проектного офиса Санкт-Петербургского государственного университета (199034, Россия, г. Санкт-Петербург, Университетская наб., 7-9, mehedova@yandex.ru)

\title{
ИСКУССТВЕННЫЙ ИНТЕЛЛЕКТ В КОНТЕКСТЕ ПУБЛИЧНОГО УПРАВЛЕНИЯ
}

\begin{abstract}
Аннотация. Работа посвящена исследованию потенциала искусственного интеллекта и проблем, связанных с его использованием в контексте публичного управления. Особое внимание уделяется вопросам этики, а именно то, как внедрить этические принципы в системы искусственного интеллекта, чтобы гарантировать, что они действуют морально. Проблема этики охватывает широкий спектр аспектов - от разработки правил искусственного интеллекта до совместимости машин и человеческих ценностей. Все возможности искусственного интеллекта имеют значительные этические последствия, которые еще только предстоит осмыслить.
\end{abstract}

Ключевые слова: публичное управление, искусственный интеллект, машинное обучение, нейронные сети, глубокое обучение, этические проблемы

$\mathrm{H}$ есмотря на то что технологии искусственного интеллекта существуют уже некоторое время, эффект от его использования в различных сферах жизни общества еще предстоит осмыслить.

В последние годы исследователи и практики инвестируют в технологические возможности искусственного интеллекта для обработки больших объемов данных государственного управления. При этом искусственный интеллект, как правило, определяется как междисциплинарная область исследований, которая открывает множество новых возможностей.

Глобальная тенденция использования технологий искусственного интеллекта во многих сферах жизни общества, включая сложные системы в области транспорта, космоса, медицины и др., вызывает многочисленные дискуссии. Ценностный потенциал искусственного интеллекта также приобретает все большее значение в контексте публичного управления [Wirtz, Weyerer, Geyer 2018: 17]. Так, Китай и США признали ценность искусственного интеллекта. Правительство Китая продемонстрировало исключительную решимость догнать западный мир в области развития искусственного интеллекта за ближайшие три года [Knight 2017: 68].

Совет Китайской Народной Республики выпустил руководство по развитию искусственного интеллекта, в котором поставлена цель стать глобальным новатором в этой области с общим объемом инвестиций в 1 трлн юаней $(147,8$ млрд долл. США) к 2030 г. ${ }^{1}$

США активно продвигают исследования и соответствующие образовательные программы, связанные с искусственным интеллектом. Например, в 2016 г. они потратили около 1,2 млрд долл. на исследования и разработки технологий, связанных с искусственным интеллектом [Preparing for the Future... 2016]. Аналогичным образом Европа потратила до 700 млн евро на робототех-

1 China issues guidelines on artificial intelligence development. - State Council of the People's Republic of China. URL: http://english.www.gov.cn/policies/latest_releases/2017/07/20/ content_281475742458322.htm (accessed 01.10.2021). 
нику и государственно-частное партнерство в области искусственного интеллекта 1 .

В то же время, несмотря на предпринятые усилия и увеличение инвестиций в исследования искусственного интеллекта, по-прежнему не ведется широкое обсуждение его использования в государственном секторе. Таким образом, по сравнению с бизнес-сферами использование искусственного интеллекта в области публичного управления все еще находится в зачаточном состоянии.

Искусственный интеллект и машинное обучение - два понятия, которые часто используются как тождественные. Однако значение этих двух терминов не одинаково. Искусственный интеллект можно определить как интеллектуальные системы, способные думать и учиться [Russell, Norvig 1995]. В настоящее время несколько технологий подпадают под определение искусственного интеллекта: нейронные сети, глубокое обучение - метод, который позволяет вычислительным моделям нескольких уровней обработки изучать и представлять данные с несколькими уровнями абстракции, имитируя то, как мозг воспринимает и понимает мультимодальную информацию [Voulodimos et al. 2018: 12]. Таким образом, машинное обучение можно рассматривать как подмножество искусственного интеллекта - машина может адаптировать свой собственный алгоритм к ситуации и «учиться», поэтому система буквально перекодирует себя [Bini 2018: 2359].

Указанные методы: компьютерное зрение - алгоритмическое обследование и анализ изображений [Jarrahi 2018]; обработка естественного языка - область исследований и приложений, изучающая, как машины могут быть использованы для понимания и анализа языкового текста или речи [Reshamwala, Mishra, Pawar 2013: 115]; распознавание речи, которое позволяет устройствам распознавать, адаптировать и переводить голосовую информацию в понятных формах. Голосовые пользовательские интерфейсы, такие как голосовой набор [Husnjak, Perakovic, Jovovic 2014: 780] достигли выдающихся результатов в решении важных проблем.

Следуя данной логике, Е. Кутергина рассматривает искусственный интеллект как пространство, населенное такими технологиями, как машинное обучение и языковая обработка. Комбинацию и применение этих технологий в направлении попыток воспроизвести или превзойти наше собственное познание можно рассматривать как искусственный интеллект [Kutergina 2017: 122].

К. Хейнонен и др. придерживаются той же точки зрения и утверждают, что искусственный интеллект имеет отношение к теории и разработке компьютерных систем, способных выполнять задачи, обычно требующие человеческого интеллекта, а машинное обучение, в свою очередь, является подмножеством искусственного интеллекта, которое часто использует статистические методы для того, чтобы дать компьютерам возможность «учиться», не будучи явно запрограммированными на это [Heinonen,Kietzmann, Pitt 2019].

Отметим, что по своему происхождению концепция искусственного интеллекта отражала намерение заменить человеческий интеллект машинами. В своем эссе «Вычислительные машины и интеллект» Алан Тьюринг предвидел, что такие проекты столкнутся с противоречиями [Turing 1950]. В этой работе он затронул вопрос, могут ли машины мыслить. Его гипотеза заключалась в том, что люди больше не смогут различать человеческий и машинный интеллект по прошествии определенного времени, и, таким образом, данный вопрос поте-

1 Ansip A. 2017. Making the most of robotics and artificial intelligence in Europe. URL: https://www.europanu.nl/id/vkjeg6jo7zyt/nieuws/blog_making_the_most_of_robotics_ and?ctx $=$ vhyzn0vt5cvu (accessed 01.10.2021). 
ряет актуальность. Однако это не произошло. Вместо этого образовались два лагеря: одни придерживались так называемого сильного тезиса, согласно которому искусственный интеллект может и будет воспроизводить человеческий интеллект, в то время как другие - сторонники «слабого тезиса» - ссылаются на способность машин эффективно решать определенные проблемы рациональным образом. Таким образом, существует фундаментальное разногласие в отношении целей и возможностей искусственного интеллекта. Однако, если цели технологий спорны, их развитие и возможные области применения не предопределены.

Актуальным остается вопрос о масштабах и границах использования искусственного интеллекта. Многие системы искусственного интеллекта уже используются в государственном управлении. Иногда искусственный интеллект способствует эволюционному развитию существующих систем. Примером являются системы управления движением.

Использование искусственного интеллекта вызвало дискуссию в науке и обществе о социальных и этических последствиях его использования в государственном секторе, а также юридических условиях, включая вопросы конфиденциальности и ответственности. Действительно, несмотря на то что правительственные организации по всему миру выступили с инициативами по внедрению и применению искусственного интеллекта, в государственном секторе внедрение технологии искусственного интеллекта представляет собой серьезную проблему [Cath et al. 2018: 520]. Так, исследователи отмечают, что большая часть реализованных с помощью искусственного интеллекта решений, «вероятно, останутся слабыми и узкоспециализированными» в течение следующих десятилетий [Cath et al. 2018: 520], что можно объяснить серьезными проблемами, которые препятствуют развитию искусственного интеллекта в государственном секторе, среди которых безопасность, качество и интеграция данных, недостаточный бюджет и др.

Безопасность выступает как важный фактор риска. В этом контексте Н. Бостром и И. Юдковски подчеркивают, что технологии искусственного интеллекта должны быть устойчивыми к неблагоприятным манипуляциям со стороны людей [Bostrom, Yudkowsky 2014: 16]. Качество системы данных и интеграция имеют большое значение, потому что система искусственного интеллекта настолько умна, насколько умны предоставленные данные, по которым она учится. Данные рассматриваются как «фундаментальный драйвер современных систем искусственного интеллекта» [Thierer, O’Sullivan Castillo, Russell 2017: 10]. Данные низкого качества или ненадежные данные также представляют собой серьезную проблему.

Финансовая осуществимость тоже играет решающую роль во внедрении технологий искусственного интеллекта. Недостаточный бюджет является одной из основных проблем, с которыми сталкиваются организации при запуске программ искусственного интеллекта.

Специализация и опыт - еще один важный аспект внедрения технологий искусственного интеллекта в государственном секторе. Стремительный рост технологий искусственного интеллекта сопровождается потребностью в специалистах и экспертах с соответствующими навыками.

Законы и нормативные акты в области искусственного интеллекта относятся к общему управлению искусственным интеллектом и, таким образом, касаются общей способности управлять и контролировать технологию искусственного интеллекта и ее социальное и экономическое влияние. Правительствам всего мира предстоит согласовать глобальные принципы и правила для систем 
искусственного интеллекта, которые должны быть основаны на стандартах демократии и прав человека [Boyd, Wilson 2017: 38].

Разработка и создание глобальной и гибкой системы управления искусственным интеллектом, которая также учитывает культурные различия и различные национальные правовые системы, чрезвычайно сложна и представляет собой серьезную проблему [Gasser, Almeida 2017: 60].

Ответственность и подотчетность тесно связаны с аспектом управления. Например, встает вопрос, кто несет ответственность, когда, например, автономное транспортное средство общественного транспорта причиняет вред пешеходу. Оборудование, разработчик программного обеспечения, поставщик или оператор, органы власти или даже само приложение? ${ }^{1}$

Несмотря на то что существуют различные подходы к решению проблемы ответственности и подотчетности в контексте использования искусственного интеллекта, в настоящее время отсутствует консенсус относительно того, как справиться с этой серьезной проблемой.

Активно обсуждаемая проблема, связанная с использованием искусственного интеллекта в публичном управлении, касается и вопросов этики.

Основные этические аспекты относятся к области этики роботов, или машинной этики. С одной стороны, это включает соображения этичности разработок и использования определенных приложений искусственного интеллекта и их последствий. С другой стороны, это связано с вопросом, как внедрить этические принципы в системы искусственного интеллекта, чтобы гарантировать, что последние действуют морально [Anderson, Anderson 2011].

Таким образом, проблема этики охватывает широкий спектр аспектов - от разработки правил искусственного интеллекта до совместимости машин и человеческих ценностей. Установление правил для поведения людей представляет собой одну из основных этических проблем, связанных с искусственным интеллектом. Системы искусственного интеллекта, как правило, предназначены для того, чтобы воспроизводить или имитировать человеческое поведение и принимать решения за людей с целью максимальной эффективности, а также минимизации ошибок, чтобы в конечном итоге принять правильное или лучшее решение за них.

Соответственно, системы искусственного интеллекта стремятся «думать и действовать рационально и воспроизводят естественный процесс принятия решений» [Banerjee, Pradeep Kumar, Bajpai 2018].

Однако, поскольку системы искусственного интеллекта не безупречны и могут иметь место неверные суждения с точки зрения человека, принятие решений на основе искусственного интеллекта может представлять угрозу для людей [Krausová 2017: 59]. Чтобы избежать ошибочных суждений, системы искусственного интеллекта полагаются на беспристрастные данные высокого качества, предоставление которых само по себе уже представляет серьезную проблему ${ }^{2}$.

Еще одна важная проблема возникает из-за того обстоятельства, что люди, в отличие от систем искусственного интеллекта, не просто действуют инструментально рационально, но также характеризуются другими чертами, которые влияют или определяют их решения и поведение, такие как сознание или эмоции [Banerjee, Pradeep, Kumar, Bajpai 2018].

${ }^{1}$ Ethically Aligned Design: A Vision for Prioritizing Human Well-Being with Autonomous and Intelligent Systems. - IEEE. 2017. URL: https://standards.ieee.org/content/dam/ieee-standards/ standards/web/documents/other/ead_v2.pdf (accessed 01.10.2021).

2 The growing impact of AI on business. 2018. URL: https://www.technologyreview. com/2018/04/30/143136/the-growing-impact-of-ai-on-business/ (accessed 01.10.2021). 
Поскольку в системах искусственного интеллекта отсутствуют эти человеческие черты, возникает вопрос и проблема, является ли этичным и морально оправданным, когда системы искусственного интеллекта устанавливают правила человеческого поведения? Кроме того, открытым остается вопрос, насколько хорошо, когда решения принимаются независимо от человеческих черт, таких как эмоции или сознание, особенно в ситуациях, когда человекоразмерность играет важную роль?

В заключение еще раз отметим, что достижения в области искусственного интеллекта действительно позволили раскрыть огромный потенциал этих новых технологий. Потенциальные преимущества технологий искусственного интеллекта огромны, но не менее велики и возникающие с их использованием риски.

\section{Список литературы}

Anderson M., Anderson S.L. 2011. Machine Ethics. N.Y., NY: Cambridge University Press.

Banerjee S., Pradeep Kumar S., Bajpai J. 2018. A Comparative Study on Decision-making Capability between Human and Artificial Intelligence. Nature Inspired Computing. P. 203-210. URL: https://www.researchgate.net/ publication/320214054_A_Comparative_Study_on_Decision-Making_Capability_ Between_Human_and_Artificial_Intelligence (accessed 20.09.2021).

Bini S. 2018. Artificial Intelligence, Machine Learning, Deep Learning, and Cognitive Computing: What Do These Terms Mean and How Will They Impact Health Care? - The Journal of Arthroplasty. Vol. 33. Is. 8. P. 2358-2361.

Bostrom N., Yudkowsky E. 2014. The Ethics of Artificial Intelligence. URL: https:// www.nickbostrom.com/ethics/artificial-intelligence.pdf (accessed 01.10.2021)

Boyd M., Wilson N. 2017. Rapid Developments in Artificial Intelligence: How Might the New Zealand Government Respond? - Policy Quarterly. Vol. 13. Is. 4. P. 36-44.

Cath C., Wachter S., Mittelstadt B., Taddeo M., Floridi L. 2018. Artificial Intelligence and the 'Good Society': The US, EU, and UK Approach. - Science and Engineering Ethics. Vol. 24. Is. 2. P. 505-528.

Gasser U., Almeida V.A.F. 2017. A Layered Model for AI Governance. - IEEE Internet Computing. Vol. 21. Is. 6. P. 58-62.

Heinonen K., Kietzmann J., Pitt L. 2019. AI and Machine Learning in Service Management. - Special issue call for papers from Journal of Service Management. URL: https://www.emeraldgrouppublishing.com/archived/products/journals/call_ for_papers.htm\%3Fid\%3D8053 (accessed 10.10.2021).

Husnjak S., Perakovic D., Jovovic I. 2014. Possibilities of Using Speech Recognition Systems of Smart Terminal Devices in Traffic Environment. - Procedia Engineering. Vol. 69. P. 778-787.

Jarrahi M. 2018. Artificial Intelligence and the Future of Work: Human-AI Symbiosis in Organizational Decision Making. - Business Horizons. Vol. 61. Is. 4. P. 577-586.

Knight W. 2017. China's AI Awakening: The West Shouldn't Fear China's ArtificialIntelligence Revolution. - Technology Review. Vol. 120. No. 6. P. 66-72.

Krausová A. 2017. Intersections between Law and Artificial Intelligence. International Journal of Computer. Vol. 27. Is. 1. P. 55-68.

Kutergina E. 2017. Computer-based Simulation Games in Public Administration Education. NISPAcee. - Journal of Public Administration and Policy. Vol. 10. Is. 2. P. 119-133.

Preparing for the Future of Artificial Intelligence. 2016. Washington, DC. Executive Office of the President National Science and Technology Council Committee 
on Technology. URL: https://obamawhitehouse.archives.gov/sites/default/files/ whitehouse_files/microsites/ostp/NSTC/preparing_for_the_future_of_ai.pdf (01.10.2021).

Reshamwala A., Mishra D., Pawar P. 2013. Review on Natural Language Processing. - IRACST Engineering Science and Technology: An International Journal (ESTIJ). Vol. 3. Is. 1. P. 113-116.

Russell S., Norvig P. 1995. Artificial Intelligence: A Modern Approach. Englewood Cliffs, NJ: Prentice-Hall. 932 p.

Thierer A., O'Sullivan Castillo A., Russell R. 2017. Artificial Intelligence and Public Policy. URL: https://www.researchgate.net/publication/319244302_Artificial_ Intelligence_and_Public_Policy (accessed 01.10.2021).

Turing A. 1950. Computing Machinery and Intelligence. URL: https://www.csee. umbc.edu/courses/471/papers/turing.pdf accessed (01.10.2021).

Voulodimos A., Doulamis N., Doulamis A., Protopapadakis E. 2018. Deep Learning for Computer Vision: a brief review. - Computational Intelligence and Neuroscience. P. 1-13. URL: https://www.researchgate.net/publication/322895764_Deep_ Learning_for_Computer_Vision_A_Brief_Review (accessed 02.10.2021).

Wirtz B., Weyerer J., Geyer C. $20 \overline{1} 8$. Artificial Intelligence and the Public Sector Applications and Challenges. - International Journal of Public Administration. Vol. 42. Is. 7. P. 1-20.

MIKHALCHENKOVA Natalia Alekseevna, Dr.Sci. (Pol.Sci.), Head of the Arctic Project Office, Saint Petersburg State University (7-9 Universitetskaya Emb, Saint-Petersburg, Russia, 199034; mehedova@yandex.ru)

\section{ARTIFICIAL INTELLIGENCE IN THE CONTEXT OF PUBLIC ADMINISTRATION}

\footnotetext{
Abstract. The article is devoted to the study of the potential and problems associated with the use of artificial intelligence in the context of public administration. Particular attention is paid to ethical issues, how to embed ethical principles in artificial intelligence systems to ensure that they act morally. The issue of ethics covers a wide range of aspects, from the development of artificial intelligence rules to the compatibility of machines and human values. All the possibilities of artificial intelligence have significant ethical implications that have yet to be comprehended.

Keywords: public administration, artificial intelligence, machine learning, neural networks, deep learning, ethical issues
} 